\title{
Higher Order Corrections to the Cross Section of
}

\author{
$e^{+} e^{-} \rightarrow$ quarkonium $+\gamma$ \\ Wen-Long Sang ${ }^{1}$ and Yu-Qi Chen ${ }^{1}$ \\ ${ }^{1}$ Key Laboratory of Frontiers in Theoretical Physics, \\ Institute of Theoretical Physics, Academia Sinica, Beijing 100190, China
}

\begin{abstract}
The cross sections of $e^{+} e^{-}$to $S$-wave and $P$-wave quarkonia with $C$-parity even associated with a photon are analyzed in the framework of non-relativistic Quantum Chromodynamics(NRQCD) factorization formulism. The short-distance coefficients are analytically determined up to the nextto-leading order(NLO) QCD radiative corrections to $S$-wave and $P$-wave quarkonium production and NLO relativistic correction to $\eta_{c}$ production. We also invoke the analytical expressions to estimate the cross sections. Our numerical results indicate that both the QCD and the relativistic corrections are considerable.
\end{abstract}

PACS numbers: 12.38.-t, 12.39.St, 13.66.Bc, 14.40.Gx 


\section{INTRODUCTION}

Non-relativistic quantum chromodynamics(NRQCD) factorization formalism[1] is an useful tool for analyzing the inclusive production of the heavy quarkonium. According to it, the cross section is expressed as a sum of products of short-distance coefficients and NRQCD matix elements. The short-distance coefficients can be calculated as perturbation series in coupling constant $\alpha_{s}$ at the scale of the heavy quark mass. The matrix elements scale in a definite way with the typical relative velocity $v$ of heavy quark in the quarkonium state. Thus the production cross sections can be expressed as double expansions both in $\alpha_{s}$ and in $v$ to any desired order.

Recently, the cross section of the exclusive process $e^{+} e^{-} \rightarrow H+\gamma$ at the center-ofmomentum(CM) energy $\sqrt{s}=10.58 \mathrm{GeV}$ was computed by Chung, Lee, and Yu[2], where $H$ represents a $S$-wave or $P$-wave heavy quarkonium state with charge-conjugation parity $C=+1$, to the leading order(LO) both in $\alpha_{s}$ and in $v$ in the NRQCD factorization formalism. Their predicted cross sections are about $82 \mathrm{fb}, 49 \mathrm{fb}, 1 \mathrm{fb}, 14 \mathrm{fb}$ and $5 \mathrm{fb}$ for $\eta_{c}, \eta_{c}(2 S), \chi_{c 0}, \chi_{c 1}$ and $\chi_{c 2}$ production, respectively. Some early calculations of similar processes like $Z^{0} \rightarrow H+\gamma$ were presented in [3] in the color-singlet model.

These relatively large cross sections make the processes be measurable at the $\mathrm{B}$ factories. To have more precise predictions, it is necessary to carry out the calculations to higher order both in $\alpha_{s}$ and in $v$. One may expect that those correction terms are considerable. Especially, for charmonium production, both $\alpha_{s}$ and $v^{2}$ are not small. In this paper, in the framework of NRQCD factorization formalism, we compute the short-distance coefficients

of the leading NRQCD matrix element to the NLO QCD corrections both for $S$-wave and $P$-wave heavy quarkonium production. For the $\eta_{c}$, we also calculate the tree level shortdistance coefficient of the $v^{2}$ relativistic correction term. We will not perform the calculation for the NLO relativistic corrections to $\eta_{c}(2 S)$ and $P$-wave quarkonium production since the NLO NRQCD matrix elements for these states are still not determined preciously by fitting data. The NLO QCD correction to the $e^{+} e^{-} \rightarrow \eta_{c}+\gamma$ was calculated first in [4]. However, we found that their original expressions given in Eqs. (23), (25) in their paper were incorrect. Probably there were some typos in their equations.

The NRQCD factorization formulism separates the short-distance effects happened at energy scale $m$ or higher, where $m$ is the heavy quark mass, and the long-distance ef- 
fects happened at long-distance $1 / m v$ or larger which describes the formation of the heavy quarkonium. The short-distance effects are described by the short-distance coefficients while the long-distance ones are described by the NRQCD long-distance matrix elements. In the process $e^{+} e^{-} \rightarrow H+\gamma$, the emission of the photon can be either short-distance effects or long-distance ones depending on the emitted photon being hard or soft comparing to the quark mass $m$. Thus when the NRQCD factorization approach is used to analyze the process of $e^{+} e^{-} \rightarrow H+\gamma$ at the B factories, the factorization formulism for the cross sections takes different forms in the cases of $H$ being $c \bar{c}$ or $b \bar{b}$ state. In the former case, the emitted photon is so hard that the short-distance process is $e^{+} e^{-} \rightarrow c \bar{c}+\gamma$. However, in the later one, the energy of the emitted photon is much less than the $b$ quark mass so that the emission of the photon is a long-distance effect and should be described by the long-distance matrix as an electric $E 1$ or a magnetic $M 1$ transition.

The rest of this article is organized as follows. In Section II, we apply the NRQCD factorization formulism to analyze $e^{+} e^{-} \rightarrow H+\gamma$ at the $\mathrm{B}$ factories where we distinguish two different cases of $c \bar{c}$ or $b \bar{b}$, corresponding to the emitted photon being hard or soft. In Section III, we determine the short-distance coefficient for the cross section of the process $e^{+} e^{-} \rightarrow \eta_{c}+\gamma$ up to the NLO radiative QCD correction for the LO matrix element and the LO short-distance coefficient for the relativistic correction term. We also discuss various limitations of those short-distance coefficients. In Section IV, we determine the short-distance coefficients for the cross sections of the processes of $e^{+} e^{-} \rightarrow \chi_{c J}+\gamma$ up to the NLO QCD radiative corrections and discuss various limitations. Section V contributes to discussions of the factorization form for the processes of $e^{+} e^{-} \rightarrow \eta_{b}\left(\chi_{b J}\right)+\gamma$ with the emitted photon being soft. In Section VI, we apply the obtained formulae to carry out numerical estimations for the cross sections of those processes. We also summarize our results in this section. We present the analytical NLO short-distance coefficients of the leading order NRQCD matrix elements in $v$ for the cross sections of $e^{+} e^{-} \rightarrow \eta_{c}+\gamma$ and $e^{+} e^{-} \rightarrow \chi_{c J}+\gamma$ in Appendix A and Appendix B, respectively.

\section{NRQCD FACTORIZATION FORMULISM FOR $e^{+} e^{-} \rightarrow H+\gamma$}

In the process of $e^{+} e^{-} \rightarrow H+\gamma$, where $H$ represents a $S$-wave or $P$-wave heavy quarkonium state with C-parity even, an almost on-shell and near threshold $Q \bar{Q}$ pair is 
created at the short-distance scale of $1 / m$ or smaller, then constitutes a heavy quarkonium $H$ that happened at the distance scale of $1 / m v$. The production cross section can be analyzed in the NRQCD factorization formulism[1] as double expansions both in velocity $v$ and in coupling $\alpha_{s}$. The factorization takes two different forms in two different cases corresponding to the emitted photon being hard and soft.

For charmonium production at the $\mathrm{B}$ factories via process $e^{+} e^{-} \rightarrow$ charmonium $+\gamma$, the momentum of the emitted photon satisfies $|k| \geq m$. The photon is so hard that the short-distance effects arise from the process $e^{+} e^{-} \rightarrow c \bar{c}+\gamma$. We assign $\mathcal{K}_{n}$ with $n$ in the collection of ${ }^{1} S_{0},{ }^{3} P_{0},{ }^{3} P_{1}$ and ${ }^{3} P_{2}$ as follows [1]:

$$
\begin{aligned}
\mathcal{K}_{{ }_{S_{0}}} & =1 \\
\mathcal{K}_{{ }^{3} P_{0}} & =\frac{1}{\sqrt{3}}\left(-\frac{i}{2} \overleftrightarrow{\boldsymbol{D}} \cdot \sigma\right), \\
\mathcal{K}_{{ }^{3} P_{1}} & =\frac{1}{\sqrt{2}}\left(-\frac{i}{2} \overleftrightarrow{\boldsymbol{D}} \times \sigma\right), \\
\mathcal{K}_{{ }^{3} P_{2}} & =-\frac{i}{2} \stackrel{\leftrightarrow}{D}^{(i} \sigma^{j)}
\end{aligned}
$$

The NRQCD factorization formulism for the cross section of $e^{+} e^{-} \rightarrow H+\gamma_{\text {hard }}\left(H=\eta_{c}\right.$, $\left.\eta_{c}(2 S), \chi_{c J}\right)$ takes the following uniform form:

$$
\begin{aligned}
\sigma & =\frac{F_{1}(n)}{m_{c}^{2}}\left\langle 0\left|\chi^{\dagger} \mathcal{K}_{n} \psi\right| H\right\rangle\left\langle H\left|\psi^{\dagger} \mathcal{K}_{n} \chi\right| 0\right\rangle \\
& +\frac{G_{1}(n)}{m_{c}^{4}} \operatorname{Re}\left\langle 0\left|\chi^{\dagger} \mathcal{K}_{n} \psi\right| H\right\rangle\left\langle H\left|\psi^{\dagger} \mathcal{K}_{n}\left(-\frac{i}{2} \overleftrightarrow{\mathbf{D}}\right)^{2} \chi\right| 0\right\rangle
\end{aligned}
$$

where $\left\langle 0\left|\chi^{\dagger} \mathcal{K}_{n} \psi\right| H\right\rangle\left\langle H\left|\psi^{\dagger} \mathcal{K}_{n} \chi\right| 0\right\rangle$ and $\operatorname{Re}\left\langle 0\left|\chi^{\dagger} \mathcal{K}_{n} \psi\right| H\right\rangle\left\langle H\left|\psi^{\dagger} \mathcal{K}_{n}\left(-\frac{i}{2} \overleftrightarrow{\mathbf{D}}\right)^{2} \chi\right| 0\right\rangle$ are NRQCD matrix elements and $F_{1}(n)$ and $G_{1}(n)$ are the short-distance coefficients corresponding to the matrix elements. The leading contributions to the matrix elements arise from these states, which possess the same quantum numbers as $n$. In (2), the NRQCD matrix element in the second term is suppressed by $v^{2}$ compared to that in the first term. The second term is usually called as the relativistic correction term. The short-distance coefficients can be expanded as power series of $\alpha_{s}$ at energy scale of $m$ or higher. They can be determined by matching the cross section of a free quark pair production process $e^{+}\left(p_{e 1}\right)+e^{-}\left(p_{e 2}\right) \rightarrow c\left(p_{1}\right) \bar{c}\left(p_{2}\right)+\gamma(k)$ with the color-singlet on-shell $c \bar{c}$ pair near the threshold. Given the total and the relative momenta of the $c \bar{c}$ pair are $P$ and $2 q$, respectively, we then have

$$
p_{1}=\frac{1}{2} P+q, \quad p_{2}=\frac{1}{2} P-q, \quad p_{1}^{2}=p_{2}^{2}=m^{2}, \quad P \cdot q=0 .
$$


Then the S-matrix element for this process is given by

$$
-i \mathcal{M}=-i \frac{e}{s} L_{\mu} \mathcal{A}^{\mu \nu} \varepsilon_{\nu}^{*}
$$

where $e$ is the electromagnetic coupling, $\varepsilon$ is the polarization four-vector of the photon and $s$ is the square of the center-of-mass $(\mathrm{CM})$ frame energy. The leptonic current $L_{\mu}$ in (3) is defined by

$$
L_{\mu}=\bar{v}\left(p_{e 2}\right) \gamma_{\mu} u\left(p_{e 1}\right)
$$

and the amplitude $\mathcal{A}^{\mu \nu}$ is given by

$$
\mathcal{A}^{\mu \nu}=\operatorname{Tr}\left[\tilde{A}^{\mu \nu} \Pi_{m}\right]
$$

where $\Pi_{m}$ is the projector operator of spin-single $(m=0)$ or spin-triplet $(m=1)$ state and $\tilde{\mathcal{A}}^{\mu \nu}$ is the amplitude of $e^{+}+e^{-} \rightarrow c \bar{c}+\gamma$ with the wave-functions of the external quark lines removed. For the spin-singlet state, $\Pi_{0}$ reads [5]

$$
\Pi_{0}=\frac{(P / 2-\not q-m) \gamma_{5}(\not P+2 E)(P / 2+\not q+m)}{4 \sqrt{6} E(E+m)}
$$

and for the spin-triplet state with spin-polarization vector $\epsilon, \Pi_{1}$ reads $[5]$

$$
\Pi_{1}=-\frac{(P / 2-\not 1-m) \not^{\star}(P+2 E)(P / 2+\not 1+m)}{4 \sqrt{6} E(E+m)},
$$

where $E=\sqrt{m^{2}+\mathbf{q}^{2}}$.

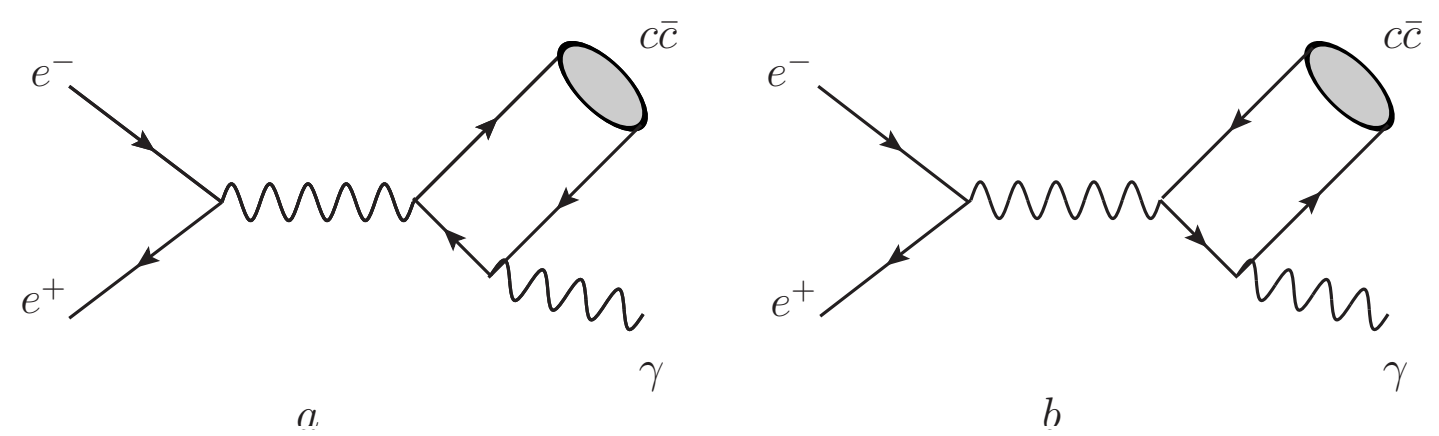

FIG. 1: The tree level Feynman diagrams for $e^{+} e^{-} \rightarrow c \bar{c}+\gamma$.

At tree level, there are two Feynman diagrams contributing to the process as illustrated in Fig. 1. $\tilde{\mathcal{A}}^{\mu \nu}$ is written as

$$
\tilde{\mathcal{A}}^{\mu \nu}=e_{Q}^{2} e^{2}\left[\gamma^{\mu} \frac{-\not p_{2}-\not k+m}{\left(p_{2}+k\right)^{2}-m^{2}} \gamma^{\nu}+\gamma^{\nu} \frac{\not p_{1}+\not k+m}{\left(p_{1}+k\right)^{2}-m^{2}} \gamma^{\mu}\right],
$$


where $e_{Q}$ is the electric charge of the heavy quark.

By expanding the amplitude in terms of $q$, the contributions arising from $S$-wave and $P$-wave can be picked out. The cross section in the QCD can then be evaluated by squaring the amplitude and integrating over the phase space. The cross section of the same process can also be evaluated in the NRQCD factorization formulism with the hadron in the NRQCD matrix elements replaced with the free quark pair being of the same quantum number as $H$. The short-distance coefficients $F_{1}(n)$ and $G_{1}(n)$ can then be inferred from equaling the cross section calculated both in the QCD and in the NRQCD factorization formulism. In our paper, we determine the $F_{1}\left({ }^{1} S_{0}\right)$ and $F_{1}\left({ }^{3} P_{J}\right)$ to the NLO QCD corrections in $\alpha_{s}$ and $G_{1}\left({ }^{1} S_{0}\right)$ to the LO in $\alpha_{s}$.

On the other hand, for bottomonium production at the $\mathrm{B}$ factories via process $e^{+} e^{-} \rightarrow$ bottomium $+\gamma$, the momentum of the emitted photon yields $|k| \ll m$. The photon is so soft that the wave-length of the emitted photon is compatible with the size of the bottomonium. Hence the emission process is sensitive to the long-distance effects. Its effects should be absorbed into the long-distance NRQCD matrix elements. The short-distance effects arise from the contributions of the process $e^{+} e^{-} \rightarrow b \bar{b}$ with $b \bar{b}$ in a spin-triplet and a color-singlet state. So, the NRQCD factorization formulism for the cross section of $e^{+} e^{-} \rightarrow H+\gamma_{\text {soft }}$ takes the following form:

$$
\begin{aligned}
\sigma & =\frac{F_{2}\left({ }^{3} S_{1}\right)}{m^{2}}\left\langle 0\left|\chi^{\dagger} \sigma^{i} \psi\right| H+\gamma\right\rangle\left\langle H+\gamma\left|\psi^{\dagger} \sigma^{i} \chi\right| 0\right\rangle \\
& +\frac{G_{2}\left({ }^{3} S_{1}\right)}{m^{4}} \operatorname{Re}\left\langle 0\left|\chi^{\dagger} \sigma^{i} \psi\right| H+\gamma\right\rangle\left\langle H+\gamma\left|\psi^{\dagger}\left(-\frac{i}{2} \overleftrightarrow{\mathbf{D}}\right)^{2} \sigma^{i} \chi\right| 0\right\rangle
\end{aligned}
$$

where $F_{2}\left({ }^{3} S_{1}\right)$ and $G_{2}\left({ }^{3} S_{1}\right)$ are the short-distance coefficients. Again, those short-distance coefficients can be inferred from perturbative QCD. The long-distance matrix elements are non-perturbative in nature.

The short-distance coefficients in this case can be determined by matching the cross section of the free quark pair production process $e^{+}+e^{-} \rightarrow b \bar{b}\left({ }^{3} S_{1}\right)$, with the $b \bar{b}$ pair near the threshold. The cross section of this process can be evaluated both in the QCD and in the NRQCD factorization formulism (9). The short-distance coefficients can then be determined by equaling the cross section inferred from both theories. The short-distance coefficients $F_{2}\left({ }^{3} S_{1}\right)$ and $G_{2}\left({ }^{3} S_{1}\right)$ in (9) have been determined to the NNLO [6] [7] and to the $\mathrm{NLO}[8]$ in the expansion of $\alpha_{s}$ in literatures. 
III. $e^{+} e^{-} \rightarrow \eta_{c}+\gamma_{h a r d}$

In this section, we apply NRQCD factorization formulism (2) to the process $e^{+} e^{-} \rightarrow$ $\eta_{c}+\gamma_{\text {hard }}$ and determine the short-distance coefficients $F_{1}\left({ }^{1} S_{0}\right)$ and $G_{1}\left({ }^{1} S_{0}\right)$ to the NLO and to the LO in $\alpha_{s}$ respectively. As mentioned in previous section, they can be determined by matching the process $e^{+}\left(p_{e 1}\right)+e^{-}\left(p_{e 2}\right) \rightarrow c\left(p_{1}\right) \bar{c}\left(p_{2}\right)\left({ }^{1} S_{0}\right)+\gamma(k)$ with $c \bar{c}$ in the color-singlet ${ }^{1} S_{0}$ state near the threshold.

Since the $c \bar{c}$ is in the ${ }^{1} S_{0}$ state, Lorentz and CPT invariance implies that the tensor $A_{\mu \nu}$ can only be expressed as

$$
\mathcal{A}^{\mu \nu}=A \epsilon_{\mu \nu \alpha \beta} P^{\alpha} k^{\beta}
$$

where $A$ is a Lorentz invariant quantity and can be inferred from perturbative QCD. Consequently, the differential and the total cross section read:

$$
\begin{aligned}
\frac{d \sigma}{d x} & =\frac{\alpha|A|^{2}}{64}\left(1-\frac{4 E^{2}}{s}\right)^{3}\left(1+x^{2}\right), \\
\sigma & =\frac{\alpha|A|^{2}}{24}\left(1-\frac{4 E^{2}}{s}\right)^{3} .
\end{aligned}
$$

Here, we use a notation $x \equiv \cos \theta$, where $\theta$ is the angle between the photon and the beam line at CM frame.

The cross section for the same process can also be derived in the NRQCD factorization formulism. Thus, we can determine the short-distance coefficients by equaling both results.

\section{A. Short-distance coefficients in the $\mathrm{LO}$ in $\alpha_{s}$}

In this subsection, we determine the short-distance coefficients $F_{1}\left({ }^{1} S_{0}\right)$ and $G_{1}\left({ }^{1} S_{0}\right)$ to the LO in $\alpha_{s}$. They can be obtained by matching the cross section of $e^{+} e^{-} \rightarrow Q \bar{Q}\left({ }^{1} S_{0}\right)+\gamma$ at tree level both in the QCD and in the NRQCD factorization formulism. To gain $G_{1}\left({ }^{1} S_{0}\right)$ which is the short-distance coefficient of the relativistic correction matrix element, we need to expand the cross section of this process to order $\mathbf{q}^{2}$.

Imposing the on-shell condition of the external quarks on (8), $\mathcal{A}^{\mu \nu}$ in (5) is simplified as

$$
\mathcal{A}^{\mu \nu}=e_{Q}^{2} e^{2} \operatorname{Tr}\left[\left(\frac{\gamma^{\nu} \not k \gamma^{\mu}}{2 p_{1} \cdot k}-\frac{\gamma^{\mu} \not k \gamma^{\nu}}{2 p_{2} \cdot k}\right) \Pi_{m}\right] .
$$

The Lorentz structure of the projection operator $\Pi_{0}$ can be written in a general form

$$
\Pi_{0}=a \gamma_{5} \not P+b \gamma_{5}(P \not-\not 1 P)+c \gamma_{5}
$$


where $a, b, c$ are Lorentz invariant and can be easily inferred from (6). One see that only the first term in (14) gives contribution to (13). Inserting this term into (13), we find

$$
\mathcal{A}^{\mu \nu}=-6 i a e_{Q}^{2} e^{2}\left(\frac{1}{p_{1} \cdot k}+\frac{1}{p_{2} \cdot k}\right) \epsilon_{\mu \nu \alpha \beta} P^{\alpha} k^{\beta} .
$$

It readily follows that

$$
A=\frac{-6 i a e_{Q}^{2} e^{2} P \cdot k}{p_{1} \cdot k p_{2} \cdot k}=\frac{-24 i a e_{Q}^{2} e^{2}}{P \cdot k}\left(1+\frac{\mathbf{q}^{2}}{3 m^{2}}\right)+O\left(\frac{\mathbf{q}^{4}}{m^{4}}\right),
$$

where we have performed a substitution[9]

$$
q^{\mu} q^{\nu} \longrightarrow \frac{1}{3}\left(-g^{\mu \nu}+\frac{P^{\mu} P^{\nu}}{4 E^{2}}\right) \mathbf{q}^{2}
$$

Meanwhile, expand $a$ with respect to $\mathbf{q}^{2}$, to obtain

$$
a=\frac{m}{2 \sqrt{6} E}=\frac{1}{2 \sqrt{6}}\left(1-\frac{1}{2} \frac{\mathbf{q}^{2}}{m^{2}}\right)+O\left(\frac{\mathbf{q}^{4}}{m^{4}}\right) .
$$

Combining (16) and (18), we present the total cross section (12) in the expansion of $\mathbf{q}^{2}$ as:

$$
\begin{aligned}
\sigma^{(0)}= & \frac{64 e_{Q}^{4} \pi^{2} \alpha^{3}}{s^{2}}(1-r) \\
& -\frac{64 e_{Q}^{4} \pi^{2}(1+2 r) \alpha^{3}}{3 s^{2}} \frac{\mathbf{q}^{2}}{m^{2}}+\mathcal{O}\left(\mathbf{q}^{\mathbf{4}}\right)
\end{aligned}
$$

where $r \equiv 4 m^{2} / s$ and superscript (0) denotes the contribution arising from the LO in $\alpha_{s}$.

Next, we turn to calculate the production cross section of $e^{+} e^{-} \rightarrow c \bar{c}\left({ }^{1} S_{0}\right)+\gamma_{\text {hard }}$ in the NRQCD factorization formulism (2). At tree level, the matrix elements give:

$$
\begin{array}{r}
\left\langle c \bar{c}\left({ }^{1} S_{0}\right)\left|\psi^{\dagger} \chi\right| 0\right\rangle=2 E \sqrt{6} \\
\left\langle c \bar{c}\left({ }^{1} S_{0}\right)\left|\psi^{\dagger}\left(-\frac{i}{2} \overleftrightarrow{\mathbf{D}}\right)^{2} \chi\right| 0\right\rangle=2 E \sqrt{6} \mathbf{q}^{2}
\end{array}
$$

Inserting them into (2) and then expanding (2) in terms of $\mathbf{q}^{2}$ up to order $\mathbf{q}^{2}$, we obtain

$$
\sigma^{(0)}=24 F_{1}^{(0)}\left({ }^{1} S_{0}\right)\left(1+\frac{\mathbf{q}^{2}}{m^{2}}\right)+24 G_{1}^{(0)}\left({ }^{1} S_{0}\right) \frac{\mathbf{q}^{2}}{m^{2}} .
$$

By equaling (21) with (19), we determine the short distance coefficients $F_{1}\left({ }^{1} S_{0}\right)$ and $G_{1}\left({ }^{1} S_{0}\right)$ to the LO in $\alpha_{s}$ :

$$
\begin{array}{r}
F_{1}^{(0)}\left({ }^{1} S_{0}\right)=\frac{8 e_{Q}^{4} \pi^{2} \alpha^{3}}{3 s^{2}}(1-r), \\
G_{1}^{(0)}\left({ }^{1} S_{0}\right)=-\frac{32 e_{Q}^{4} \pi^{2} \alpha^{3}}{9 s^{2}}\left(1-\frac{r}{4}\right) .
\end{array}
$$


Finally, the cross section of $e^{+} e^{-} \rightarrow \eta_{c}+\gamma$ to the LO in $\alpha_{s}$ and the NLO in $v^{2}$ is given as:

$$
\begin{aligned}
\sigma^{(0)} & =\frac{8 e_{Q}^{4} \pi^{2} \alpha^{3}}{3 m^{2} s^{2}}(1-r)\left\langle 0\left|\chi^{\dagger} \psi\right| \eta_{c}\right\rangle\left\langle\eta_{c}\left|\psi^{\dagger} \chi\right| 0\right\rangle \\
& -\frac{32 e_{Q}^{4} \pi^{2} \alpha^{3}}{9 m^{4} s^{2}}\left(1-\frac{r}{4}\right) \operatorname{Re}\left[\left\langle 0\left|\chi^{\dagger} \psi\right| \eta_{c}\right\rangle\left\langle\eta_{c}\left|\psi^{\dagger}\left(-\frac{i}{2} \overleftrightarrow{\mathbf{D}}\right)^{2} \chi\right| 0\right\rangle\right] .
\end{aligned}
$$

Here the two hadron matrix elements are normalized relativistically, which is related to the standard non-relativistic normalized hadron matrix elements[10] by

$$
\begin{aligned}
\left\langle 0\left|\chi^{\dagger} \psi\right| \eta_{c}\right\rangle & =\sqrt{4 m}\left(\left\langle 0\left|\chi^{\dagger} \psi\right| \eta_{c}\right\rangle_{B B L}+\frac{1}{4 m^{2}} \operatorname{Re}\left[\left\langle 0\left|\chi^{\dagger}\left(-\frac{i}{2} \overleftrightarrow{\mathbf{D}}\right)^{2} \psi\right| \eta_{c}\right\rangle_{B B L}\right]\right) \\
\left\langle 0\left|\chi^{\dagger}\left(-\frac{i}{2} \overleftrightarrow{\mathbf{D}}\right)^{2} \psi\right| \eta_{c}\right\rangle & =\sqrt{4 m}\left\langle 0\left|\chi^{\dagger}\left(-\frac{i}{2} \overleftrightarrow{\mathbf{D}}\right)^{2} \psi\right| \eta_{c}\right\rangle_{B B L}
\end{aligned}
$$

Inserting (24) into (23), we obtain the cross section with the hadron matrix elements in non-relativistic normalization as

$$
\begin{aligned}
\sigma^{(0)} & =\frac{32 e_{Q}^{4} \pi^{2} \alpha^{3}}{3 m s^{2}}(1-r)\left\langle 0\left|\chi^{\dagger} \psi\right| \eta_{c}\right\rangle\left\langle\eta_{c}\left|\psi^{\dagger} \chi\right| 0\right\rangle_{B B L} \\
& -\frac{16 e_{Q}^{4} \pi^{2} \alpha^{3}}{9 m^{3} s^{2}}(5+r) \operatorname{Re}\left[\left\langle 0\left|\chi^{\dagger} \psi\right| \eta_{c}\right\rangle\left\langle\eta_{c}\left|\psi^{\dagger}\left(-\frac{i}{2} \overleftrightarrow{\mathbf{D}}\right)^{2} \chi\right| 0\right\rangle_{B B L}\right]
\end{aligned}
$$

\section{B. The NLO QCD correction}
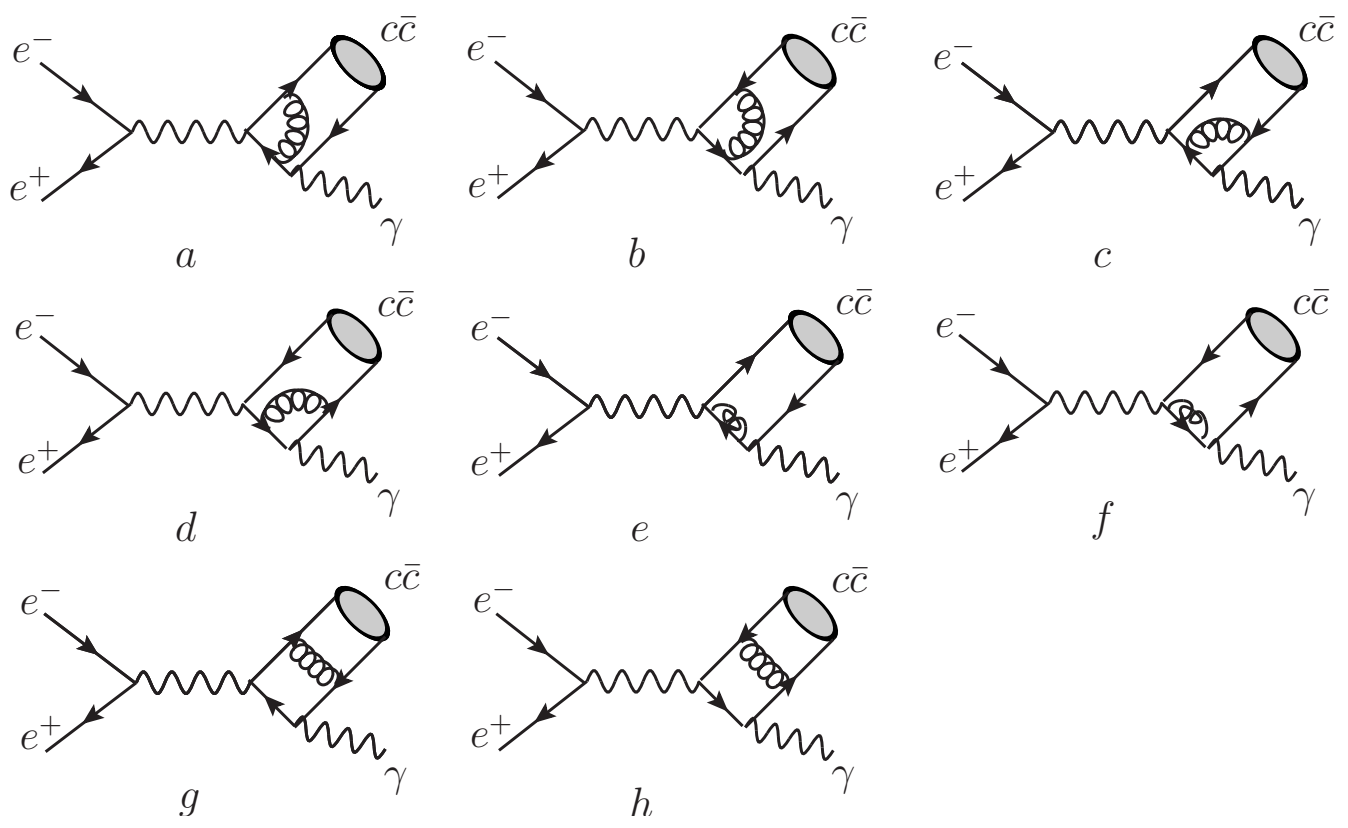

FIG. 2: One-loop Feynman diagrams for $e^{+} e^{-} \rightarrow c \bar{c}+\gamma$. 
In this subsection, we determine the short-distance coefficients $F_{1}\left({ }^{1} S_{0}\right)$ to the NLO in $\alpha_{s}$. To this end, we need to match the cross section of the process $e^{+} e^{-} \rightarrow c \bar{c}\left({ }^{1} S_{0}\right)+\gamma_{\text {hard }}$ to the one-loop level but the LO in $v^{2}$ both in the QCD and in the NRQCD factorization formulism.

The Feynman diagrams responsible for the one-loop QCD correction are illustrated in Fig. 2, We perform the renormalization in on-mass-shell(OS) scheme and take dimensional regularization to regulate both the ultraviolet(UV) and the infrared(IR) divergences. In OS scheme, external quark lines do not receive any QCD corrections, and the counterterms from the renormalization constants of the heavy quark wavefunction and the heavy quark mass are given by[11]:

$$
\begin{aligned}
\delta Z_{Q}^{\mathrm{OS}} & =-C_{F} \frac{\alpha_{s}}{4 \pi}\left(\frac{1}{\epsilon_{\mathrm{UV}}}+\frac{2}{\epsilon_{\mathrm{IR}}}-3 \gamma_{E}+3 \ln \frac{4 \pi \mu^{2}}{m_{c}^{2}}+4\right)+\mathcal{O}\left(\alpha_{s}^{2}\right), \\
\delta Z_{m Q}^{\mathrm{OS}} & =-C_{F} \frac{\alpha_{s}}{4 \pi}\left(\frac{3}{\epsilon_{\mathrm{UV}}}-3 \gamma_{E}+3 \ln \frac{4 \pi \mu^{2}}{m_{c}^{2}}+4\right)+\mathcal{O}\left(\alpha_{s}^{2}\right)
\end{aligned}
$$

where $\mu$ is the renormalization scale, $\gamma_{E}$ is the Euler's constant, and $C_{F}=\frac{4}{3}$ for $S U(3)_{c}$.

Among the eight diagrams in Fig. 2, there are two self-energy diagrams, four triangle diagrams and two box diagrams. The divergences of these diagrams can be analyzed as follows: the self-energy and the triangle diagrams contain UV divergences, while the box diagrams possess IR divergences. In addition, Coulomb singularities may arise from the box diagrams, due to the exchange of longitudinal gluon between $c$ and $\bar{c}$. Our calculations indicate that the UV divergences from the self-energy and the triangle diagrams are canceled by that from the renormalization constants of the quark field and the mass and IR divergences from the box diagrams are canceled by that in $\delta Z_{Q}$. As one will see, the remaining Coulomb singularities will be canceled by Coulomb singularities in NRQCD matrix elements by matching condition. In our practical calculations, the 'Feycalc' package[12] has been employed to take the trace of the $\gamma$-matrix and to reduce tensor integrals into scalar ones. Furthermore, we carry out the IR-safe scalar integrals by using G.'t Hooft and M. Veltman's method[13]. For the IR-divergent scalar integrals, we take the results from Ref. [14]. After canceling all these UV and IR divergences, we present the NLO cross section of $e^{+} e^{-} \rightarrow c \bar{c}\left({ }^{1} S_{0}\right)+\gamma$ :

$$
\sigma=\left.\sigma^{(0)}\right|_{\mathbf{q}=0}\left(1+\frac{\pi^{2}}{v} C_{F} \frac{\alpha_{s}}{\pi}+\frac{\alpha_{s}}{\pi} C(r)\right),
$$

where $\left.\sigma^{(0)}\right|_{\mathbf{q}=0}$ is the LO cross section (19) with $\mathbf{q}=0$ and $C(r)$ is a function of $r$ and is both UV and IR finite. The explicit expression for $C(r)$ is given in Appendix $\mathrm{A}$. 
Subsequently, we turn to deal with the same process in the NRQCD factorization formulism. The main ingredient of this calculation is to obtain the NRQCD matrix element to the NLO in $\alpha_{s}$, which reads[1],

$$
\left|\left\langle c \bar{c}\left({ }^{1} S_{0}\right)\left|\psi^{\dagger} \chi\right| 0\right\rangle\right|^{2}=24 m^{2}\left(1+\frac{\pi^{2}}{v} C_{F} \frac{\alpha_{s}}{\pi}\right)
$$

where the Coulomb singularity term emerges from the Coulomb gluon exchanging between the quark and the anti-quark. The total cross section of $e^{+} e^{-} \rightarrow c \bar{c}\left({ }^{1} S_{0}\right)+\gamma$ in the NRQCD factorization formulism can be readily expressed as

$$
\sigma=24 F_{1}\left({ }^{1} S_{0}\right)\left(1+\frac{\pi^{2}}{v} C_{F} \frac{\alpha_{s}}{\pi}\right) .
$$

By equaling (28) with (30), we determine the NLO short-distance coefficient:

$$
F_{1}\left({ }^{1} S_{0}\right)=F_{1}^{(0)}\left({ }^{1} S_{0}\right)\left(1+\frac{\alpha_{s}}{\pi} C(r)\right)
$$

from which we see that the Coulomb singularity cancels in the short-distance coefficient as expected. $1+\frac{\alpha_{s}}{\pi} C(r)$ is usually referred to the $K$-factor.

Inserting this coefficient into (2), we reach the final result for the cross section to the NLO QCD correction in $\alpha_{s}$ and the LO in $v^{2}$ :

$$
\sigma=\sigma^{(0)}\left(1+\frac{\alpha_{s}}{\pi} C(r)+\mathcal{O}\left(\alpha_{s}^{2}\right)\right)
$$

where $\sigma^{(0)}$ is given by (23) with $\mathbf{q}=0$.

It is instructive to look at the behaviors of $C(r)$ in two limits of $r \rightarrow 0$ and $r \rightarrow \infty$. The limit of $r \rightarrow 0$ corresponds to a very high energy production of $\eta_{c}$. In this limit, $C(r)$ reduces to

$$
\lim _{r \rightarrow 0} C(r)=-\frac{2}{9}\left[(9-6 \log 2) \log r+9\left(3+\log ^{2} 2-3 \log 2\right)+\pi^{2}\right]
$$

The coefficient of $\log r$ term agrees with that obtained in Ref. [15].

The limit of $r \rightarrow \infty$ corresponds to the NLO QCD corrections to the process of $\eta_{c}$ decay into two photons. To achieve this limit, one needs to recalculate the $K$-factor for $r>1$. We define $C^{\prime}(r)$ in this region via

$$
\frac{\sigma\left(r^{\star} \rightarrow \eta_{Q} \gamma\right)}{\sigma^{(0)}\left(r^{\star} \rightarrow \eta_{Q} \gamma\right)}=1+\frac{\alpha_{s}}{\pi} C^{\prime}(r),
$$


whose expression is given in Appendix A, With the expression of $C^{\prime}(r)$ in hand, we take the limit of $r \rightarrow \infty$ for $C^{\prime}(r)$, which reads

$$
\lim _{r \rightarrow \infty} C^{\prime}(r)=C_{F}\left(-5+\frac{\pi^{2}}{4}\right) .
$$

It is encouraging that (35) is consistent with the QCD correction to the cross section of $\eta_{c} \rightarrow \gamma \gamma$. Comparing our results with that given in Ref. [4], we found that the expressions given in their Eqs. (23) (25), from which they obtained the two limits of $r \rightarrow 0$ and $r \rightarrow \infty$,

were incorrect. Actually, it is easy to check that the results given in their Eqs. (23) (25) go to infinity by taking these two limits. Therefore we claim that there might be some typos in their results.

IV. $\quad e^{+} e^{-} \rightarrow \chi_{c J}+\gamma_{\text {hard }}$

In this section, we apply the NRQCD factorization formulism (2) to the processes $e^{+} e^{-} \rightarrow$ $\chi_{c J}+\gamma_{\text {hard }}$ with $J=0,1,2$ and determine the short-distance coefficients $F_{1}\left({ }^{3} P_{J}\right)$ to the NLO in $\alpha_{s}$. As mentioned in Sec. III, these coefficients can be determined by matching the processes $e^{+}+e^{-} \rightarrow c \bar{c}\left({ }^{3} P_{J}\right)+\gamma$ with the $c \bar{c}$ near the threshold and in the color-singlet ${ }^{3} P_{J}$ states.

\section{A. The LO short-distance coefficients in $\alpha_{s}$}

We first determine the LO coefficients in $\alpha_{s}$ by calculating the cross sections of $e^{+}\left(p_{e 1}\right)+e^{-}\left(p_{e 2}\right) \rightarrow c\left(p_{1}\right) \bar{c}\left(p_{2}\right)\left({ }^{3} P_{J}\right)+\gamma(k)$ both in the QCD and in the NRQCD factorization formulism. Two tree-level diagrams contributing to the processes $e^{+}\left(p_{e 1}\right)+e^{-}\left(p_{e 2}\right) \rightarrow$ $c\left(p_{1}\right) \bar{c}\left(p_{2}\right)\left({ }^{3} P_{J}\right)+\gamma(k)$ are illustrated in Fig. 1. To carry out the calculations, we need to construct the polarization vectors and tensors for $\chi_{1}$ and $\chi_{2}$ respectively. Three polarization vectors $\epsilon_{\{0, \pm 1\}}^{\mu}$ for $\chi_{1}$ can be explicitly expressed as(provided the hadron moving along $z$ axis.)

$$
\epsilon_{+1}^{\mu}=\frac{1}{\sqrt{2}}(0,-1,-i, 0), \quad \epsilon_{-1}^{\mu}=\frac{1}{\sqrt{2}}(0,+1,-i, 0), \quad \epsilon_{0}^{\mu}=\frac{1}{2 m}\left(|\mathbf{P}|, 0,0, E_{P}\right) .
$$

Then five polarization tensors for $\chi_{2}$ are readily written in terms of $\epsilon_{\{0, \pm 1\}}^{\mu}$ as

$$
\begin{aligned}
& \epsilon_{ \pm 2}^{\mu \nu}=\epsilon_{ \pm 1}^{\mu} \epsilon_{ \pm 1}^{\nu}, \quad \epsilon_{ \pm 1}^{\mu \nu}=\frac{1}{\sqrt{2}}\left(\epsilon_{ \pm 1}^{\mu} \epsilon_{0}^{\nu}+\epsilon_{0}^{\mu} \epsilon_{ \pm 1}^{\nu}\right), \\
& \epsilon_{0}^{\mu \nu}=\frac{1}{\sqrt{6}}\left(\epsilon_{+1}^{\mu} \epsilon_{-1}^{\nu}+2 \epsilon_{0}^{\mu} \epsilon_{0}^{\nu}+\epsilon_{-1}^{\mu} \epsilon_{+1}^{\nu}\right) .
\end{aligned}
$$


The amplitudes of $e^{+} e^{-} \rightarrow c \bar{c}\left({ }^{3} P_{J}\right)+\gamma$ can be picked out from (13) by extracting the contributions corresponding to the $c \bar{c}\left({ }^{3} P_{J}\right)$ production. To this end, one needs to expand the amplitudes to the LO in $q$ and to project it into the ${ }^{3} P_{J}$ states with helicity $\lambda_{H}$. It can be done by using the formulae[16] [17]:

$$
\mathcal{A}^{\alpha \beta}\left(\lambda_{H}\right)=|\mathbf{q}| \frac{\partial}{\partial q^{\mu}} \operatorname{Tr}\left[\tilde{\mathcal{A}}^{\alpha \beta} \Pi_{1 \nu}\right] \mathcal{P}_{J}^{\mu \nu},
$$

where $\Pi_{1}^{\nu}$ is defined via $\Pi_{1} \equiv \Pi_{1}^{\nu} \epsilon^{\nu}$ and the projection operators $\mathcal{P}_{J}^{\mu \nu}$ are defined by

$$
\begin{aligned}
& \mathcal{P}_{0}^{\mu \nu}=\frac{1}{\sqrt{3}}\left(-g^{\mu \nu}+\frac{P^{\mu} P^{\nu}}{4 E^{2}}\right), \\
& \mathcal{P}_{1}^{\mu \nu}=\frac{i}{2 \sqrt{2} E} \epsilon^{\mu \nu \rho \sigma} P_{\rho} \epsilon_{\lambda_{H} \sigma}^{*} \\
& \mathcal{P}_{2}^{\mu \nu}=\epsilon_{\lambda_{H}}^{* \mu \nu} .
\end{aligned}
$$

With these formulae, it is straightforward to calculate the cross sections of the processes $e^{+} e^{-} \rightarrow c \bar{c}\left({ }^{3} P_{J}\left(\lambda_{H}\right)+\gamma\right.$ with a specific helicity $\lambda_{H}$ for $c \bar{c}$. The differential cross sections of these processes in the LO in $\alpha_{s}$ yield

$$
\frac{d \sigma^{(0)}}{d x}=\frac{48 e_{Q}^{4} \alpha^{3} \pi^{2}}{s^{2} m^{2}(1-r)}|\mathbf{q}|^{2} \widetilde{F}_{J}\left(\lambda_{H}\right)
$$

where the functions $\widetilde{F}_{J}\left(\lambda_{H}\right)$ are

$$
\begin{aligned}
\widetilde{F}_{0}(0) & =\frac{(1-3 r)^{2}}{6}\left(1+x^{2}\right), \\
\widetilde{F}_{1}(0) & =\left(1+x^{2}\right), \\
\widetilde{F}_{1}( \pm 1) & =r\left(1-x^{2}\right), \\
\widetilde{F}_{2}(0) & =\frac{1}{3}\left(1+x^{2}\right), \\
\widetilde{F}_{2}( \pm 1) & =r\left(1-x^{2}\right), \\
\widetilde{F}_{2}( \pm 2) & =r^{2}\left(1+x^{2}\right) .
\end{aligned}
$$

Since relativistic corrections of ${ }^{3} P_{J}$ production will not be calculated, we have set $M_{H}=$ $2 E=2 m$. Our formulae (40) agree with that obtained in Ref. [2] [18].

We now turn to evaluate the cross sections of the same processes in the NRQCD factorization formulism (2). The matrix elements for $c \bar{c}\left({ }^{3} P_{J}\right)$ production are given by

$$
\left\langle c \bar{c}\left({ }^{3} P_{J}\left(\lambda_{H}\right)\right)\left|\psi^{\dagger}\left(\mathcal{K}_{3 P_{J}} \cdot \epsilon_{J}\right) \chi\right| 0\right\rangle=2 \sqrt{6} m|\mathbf{q}| \cdot
$$


Thus, the differential cross sections over $x$ within the framework of NRQCD factorization formulism read

$$
\frac{d \sigma^{(0)}}{d x}=24 F_{1}^{(0)}\left({ }^{3} P_{J}\left(\lambda_{H}\right)\right)|\mathbf{q}|^{2}
$$

where $F_{1}^{(0)}\left({ }^{3} P_{J}\left(\lambda_{H}\right)\right)$ denote the LO short-distance coefficients. By equaling the cross sections on the QCD side with that on the NRQCD side, we determine the short-distance coefficients:

$$
F_{1}^{(0)}\left({ }^{3} P_{J}\left(\lambda_{H}\right)\right)=\frac{2 e_{Q}^{4} \alpha^{3} \pi^{2}}{m^{2} s^{2}(1-r)} \widetilde{F}_{J}\left(\lambda_{H}\right) .
$$

Consequently, the differential cross sections of $e^{+} e^{-} \rightarrow \chi_{c J}\left(\lambda_{H}\right) \gamma$ in the LO in $v$ and $\alpha_{s}$ are written as:

$$
\frac{d \sigma^{(0)}}{d x}=\frac{F_{1}^{(0)}\left({ }^{3} P_{J}\right)}{m^{2}}\left\langle 0\left|\chi^{\dagger} \mathcal{K}_{J} \psi\right| \chi_{J}\left(\lambda_{H}\right)\right\rangle\left\langle\chi_{J}\left(\lambda_{H}\right)\left|\psi^{\dagger} \mathcal{K}_{J} \chi\right| 0\right\rangle,
$$

where the $\chi_{J}$ states in the matrix element are normalized relativistically. To express our formulae as the product of short-distance coefficients and standard non-relativistic normalized matrix elements, we can use the relations between relativistic normalized matrix elements and non-relativistic normalized matrix elements[10]

$$
\left\langle 0\left|\chi^{\dagger} \mathcal{K}_{3 P_{J}} \psi\right| H\right\rangle=\sqrt{2 M_{H}}\left\langle 0\left|\chi^{\dagger} \mathcal{K}_{{ }^{3} P_{J}} \psi\right| H\right\rangle_{B B L},
$$

where $|H\rangle_{B B L}$ is the non-relativistic normalized matrix element used in Ref. [1] and $M_{H}$ is the mass of $H$. In the LO in $v$, we set $M_{H}=\left.2 E\right|_{q=0}=2 m$.

The differential cross sections of $e^{+} e^{-} \rightarrow \chi_{c J}\left(\lambda_{H}\right)+\gamma$ can be reexpressed as:

$$
\frac{d \sigma^{(0)}}{d x}=\frac{8 e_{Q}^{4} \alpha^{3} \pi^{2}}{m^{3} s^{2}(1-r)} \widetilde{F}_{J}\left(\lambda_{H}\right)\left\langle 0\left|\chi^{\dagger} \mathcal{K}_{J} \psi\right| \chi_{c J}\right\rangle\left\langle\chi_{c J}\left(\lambda_{H}\right)\left|\psi^{\dagger} \mathcal{K}_{J} \chi\left(\lambda_{H}\right)\right| 0\right\rangle_{B B L}
$$

The total cross sections are achieved by integrating over $x$ and summing over all the helicity states $\lambda_{H}$ of the $\chi_{J}$. We present the final results:

$$
\sigma^{(0)}\left(\chi_{c J}\right)=F_{J}^{(0)}\left\langle 0\left|\chi^{\dagger} \mathcal{K}_{J} \psi\right| \chi_{c J}\right\rangle\left\langle\chi_{c J}\left|\psi^{\dagger} \mathcal{K}_{J} \chi\right| 0\right\rangle_{B B L}
$$

where $F_{J}^{(0)}$ for $J=0,1,2$ are given by

$$
\begin{aligned}
& F_{0}^{(0)}=\frac{32 e_{Q}^{4} \alpha^{3} \pi^{2}(1-3 r)^{2}}{9 m^{3} s^{2}(1-r)}, \\
& F_{1}^{(0)}=\frac{64 e_{Q}^{4} \alpha^{3} \pi^{2}(1+r)}{3 m^{3} s^{2}(1-r)}, \\
& F_{2}^{(0)}=\frac{64 e_{Q}^{4} \alpha^{3} \pi^{2}\left(1+3 r+6 r^{2}\right)}{9 m^{3} s^{2}(1-r)} .
\end{aligned}
$$




\section{B. Short-distance coefficients in the NLO in $\alpha_{s}$}

In this subsection, we determine the short-distance coefficients in the NRQCD factorization formulism for processes $e^{+} e^{-} \rightarrow \chi_{c J}+\gamma$ up to the NLO in $\alpha_{s}$. As done in the case of $\eta_{c}$ production, we calculate the cross sections of the processes $e^{+} e^{-} \rightarrow c \bar{c}\left({ }^{3} P_{J}\right)+\gamma$ both in the QCD and in the NRQCD factorization formulism to one-loop level. Feynman diagrams responsible for the processes are the same as that of ${ }^{1} S_{0}$ production as illustrated in Fig. 2. We still carry the renormalization in on-mass-shell(OS) scheme. The wave function renormalization constant and mass renormalization constant have been given in (26) (27).

The UV and IR divergences of each diagram are similar with that of $\eta_{c}$ production. The self-energy and triangle diagrams contain ultraviolet(UV) divergences. The box diagrams have both infrared(IR) divergences and Coulomb singularities. Combining contributions from the eight diagrams and the counterterms, all divergences cancel except Coulomb singularities which will be canceled by Coulomb singularities in NRQCD matrix elements. The cross sections of the processes $e^{+} e^{-} \rightarrow c \bar{c}\left({ }^{3} P_{J}, \lambda_{H}\right)+\gamma$ in the NLO in $\alpha_{s}$ are given by

$$
\frac{d \sigma}{d x}=\frac{48 e_{Q}^{4} \alpha^{3} \pi^{2}}{m^{2} s^{2}(1-r)}|\mathbf{q}|^{2} \widetilde{F}_{J}\left(\lambda_{H}\right)\left(1+\frac{\pi^{2}}{v} C_{F} \frac{\alpha_{s}}{\pi}+\frac{\alpha_{s}}{\pi} C_{J}^{\lambda_{H}}(r)\right),
$$

where the index $\lambda_{H}$ denotes the helicity of $\chi_{c J}, \widetilde{F}_{J}\left(\lambda_{H}\right)$ have been given in previous subsection and the expressions for $C_{J}^{\lambda_{H}}(r)$ are listed in Appendix $\mathrm{B}$.

One can immediately get the total cross sections by integrating over $x$ and summing over the polarizations of $\chi_{c J}$. As a result, the cross sections yield

$$
\sigma=F_{J}^{(0)}|\mathbf{q}|^{2}\left(1+\frac{\pi^{2}}{v} C_{F} \frac{\alpha_{s}}{\pi}+\frac{\alpha_{s}}{\pi} C_{J}(r)\right)
$$

where $C_{J}(r)$ for $J=0,1,2$ are given by

$$
\begin{aligned}
& C_{0}(r)=C_{0}^{0}(r), \\
& C_{1}(r)=\frac{C_{1}^{0}(r)+r C_{1}^{1}(r)}{1+r}, \\
& C_{2}(r)=\frac{C_{2}^{0}(r)+3 r C_{2}^{1}(r)+6 r^{2} C_{2}^{2}(r)}{1+3 r+6 r^{2}} .
\end{aligned}
$$

We now calculate the cross sections of the same processes in the NRQCD factorization formulism. The NRQCD matrix elements of $P$-wave operators in the LO in $v$ and NLO in $\alpha_{s}$ are given by

$$
\left|\left\langle c \bar{c}\left({ }^{3} P_{0}\right)\left|\mathcal{K}_{3 P_{J}}\right| 0\right\rangle\right|^{2}=24 m^{2}|\mathbf{q}|^{2}\left(1+\frac{\pi^{2}}{v} C_{F} \frac{\alpha_{s}}{\pi}\right) .
$$


With it, one readily obtains the cross sections of $e^{+} e^{-} \rightarrow c \bar{c}\left({ }^{3} P_{J}\right)+\gamma$ on the NRQCD side:

$$
\sigma=24 F_{1}\left({ }^{3} P_{J}\right)|\mathbf{q}|^{2}\left[1+\frac{\pi^{2}}{v} C_{F} \frac{\alpha_{s}}{\pi}\right]
$$

The short-distance coefficients $F_{1}\left({ }^{3} P_{J}\right)$ can be determined by equaling (51) with (54),

$$
F_{1}\left({ }^{3} P_{J}\right)=F_{1}^{(0)}\left({ }^{3} P_{J}\right)\left[1+\frac{\alpha_{s}}{\pi} C_{J}(r)\right]
$$

Finally, the cross sections of the processes $e^{+} e^{-} \rightarrow \chi_{c J}+\gamma_{h a r d}$ in the NLO in $\alpha_{s}$ can be expressed as

$$
\sigma\left(\chi_{c J}\right)=\sigma^{(0)}\left(\chi_{c J}\right)\left(1+\frac{\alpha_{s}}{\pi} C_{J}(r)\right)
$$

where $\sigma_{c J}^{(0)}$ denote the LO cross sections given in (48).

It is interesting to look at the behaviors of $C_{J}(r)$ in two limits of $r \rightarrow 0$ and $r \rightarrow \infty$. The limit of $r \rightarrow 0$ is related to the $\chi_{c J}$ production in a very high energy. In this limit, we find

$$
\begin{aligned}
& \lim _{r \rightarrow 0} C_{0}(r)=-\frac{2}{9}\left[(3-6 \log 2) \log r+9 \log ^{2} 2-33 \log 2+\pi^{2}\right], \\
& \lim _{r \rightarrow 0} C_{1}(r)=-\frac{2}{9}\left[(9-6 \log 2) \log r+9 \log ^{2} 2-15 \log 2+\pi^{2}+21\right], \\
& \lim _{r \rightarrow 0} C_{2}(r)=-\frac{2}{9}\left[(3-6 \log 2) \log r+9 \log ^{2} 2+3 \log 2+\pi^{2}+18\right] .
\end{aligned}
$$

The limit of $r \rightarrow \infty$ is related to the NLO QCD corrections to the cross sections of $\chi_{c J} \rightarrow \gamma \gamma$. As in the case of $\eta_{c}$, one needs to recalculate the $K$-factor for $r>1$. We define $C_{c J}^{\prime}(r)$ via

$$
\frac{\sigma\left(r^{\star} \rightarrow \chi_{J} \gamma\right)}{\sigma^{0}\left(r^{\star} \rightarrow \chi_{J} \gamma\right)}=1+\frac{\alpha_{s}}{\pi} C_{J}^{\prime}(r)
$$

Explicit expressions of $C_{c J}^{\prime}(r)$ are listed in Appendix B, In the limit of $r \rightarrow \infty, C_{J}^{\prime}(r)$ go to:

$$
\begin{aligned}
& \lim _{r \rightarrow \infty} C_{0}^{\prime}=C_{F}\left(-\frac{7}{3}+\frac{\pi^{2}}{4}\right), \\
& \lim _{r \rightarrow \infty} C_{2}^{\prime}=-4 C_{F} .
\end{aligned}
$$

The formulae(59) are desirable, since these are nothing but the QCD corrections to the processes $\chi_{0,2} \rightarrow \gamma \gamma$. In (59), we did not take the limit of $r \rightarrow \infty$ for $C_{1}^{\prime}(r)$, since the cross section of $\chi_{c 1}$ decay into $\gamma \gamma$ vanishes due to the Yang's theory. 
V. $\quad e^{+} e^{-} \rightarrow \eta_{b}\left(\chi_{b J}\right)+\gamma_{s o f t}$

As discussed in Sec. II, for bottomonium production at the B factories through process $e^{+}+e^{-} \rightarrow$ bottomonium $+\gamma$, the emitted photon is soft comparing to the $b$ quark mass. The cross section of the process is expressed as (9) in the framework of the NRQCD factorization formulism. The short-distance coefficients $F_{2}\left({ }^{3} S_{1}\right)$ and $G_{2}\left({ }^{3} S_{1}\right)$ in (9) can be determined by matching the cross section of the process $e^{+}\left(p_{e 1}\right)+e^{-}\left(p_{e 2}\right) \rightarrow c\left(p_{1}\right) \bar{c}\left(p_{2}\right)$ with the $c \bar{c}$ in the color-singlet ${ }^{3} S_{1}$ state predicted by QCD and by the NRQCD factorization formulism. It has been calculated up to order $\alpha_{s}^{2}$. Here we list only the $F_{2}\left({ }^{3} S_{1}\right)$ result [5] [6] [7] [19] [20]:

$$
F_{2}\left({ }^{3} S_{1}\right)=\frac{4 \pi^{3} e_{Q}^{2} \alpha^{2} \delta\left(s-4 m^{2}\right)}{m}\left\{1-4 C_{F} \frac{\alpha_{s}}{\pi}+\left[-117.46+0.82 n_{f}+\frac{140 \pi^{2}}{27} \log \frac{2 m}{\mu}\right] \frac{\alpha_{s}^{2}}{\pi^{2}}\right\}
$$

The matrix elements in (9) describe probabilities of a point-like ${ }^{3} S_{1} b \bar{b}$ state transiting into the ${ }^{1} S_{0}$ or the ${ }^{3} P_{J}$ state through a magnetic $M 1$ transition or an electric $E 1$ transition by emitting one soft photon. Although they are QED processes, the matrix elements are non-perturbative in nature. One may evaluate those $M 1$ and $E 1$ transition processes by perturbative QCD and relate the matrix elements appearing in (9) to those appearing in (2). However, this kind of calculations is not reliable. Some additional Coulomb singularities may arise from gluon exchange between almost on-shell $b$ and $\bar{b}$ quarks. Terms like $\alpha_{s} / v$ appearing in radiative corrections spoil fixed order perturbation calculations on those matrix elements. Even at tree level, it is proportional to a factor $s / 4 m^{2}-1$ for the $\eta_{b}$ production and $1 /\left(s / 4 m^{2}-1\right)$ for the $\eta_{c J}$ production. Thus the results are very sensitive to the quark mass. To gain a reliable prediction on the matrix in (9), one need to invoke some nonperturbative methods such as lattice QCD.

\section{NUMERICAL RESULTS AND DISCUSSION}

In this section, we carry our numerical calculations on the production cross sections of $e^{+} e^{-} \rightarrow H+\gamma\left(H=\eta_{c}, \eta_{c}(2 S), \chi_{c J}\right)$ at the $\mathrm{B}$ factories using the results derived in section III and section IV. To this end, we need to determine some relevant parameters such as quark mass, $\alpha_{s}(\mu)$, and those NRQCD matrix elements. We will estimate them below. 


\section{A. Input parameters}

At the $\mathrm{B}$ factories, we take $\sqrt{s}=10.6 \mathrm{GeV}$ and $\alpha(\mu=\sqrt{s})=1 / 131$. The quark mass is taken as $m_{c}=1.4 \mathrm{GeV}$. There is an ambiguity in choosing the value of $\mu$ in $\alpha_{s}$ since there are several energy scales involved, such as $\sqrt{s}$ and heavy quark mass. In the limit of $r \rightarrow 0$, one can use the leading log approximation (LLA) to resume those large logarithmic terms such as $\left(\alpha_{s}(\sqrt{s}) \ln \sqrt{s} / m\right)^{n}[4,15]$. This accounts for the effects of the running of the strong coupling constant $\alpha_{s}$ from energy scale $\sqrt{s}$ to $m$. However, in doing this approximation, one has omitted constant terms and terms suppressed by power of $r$. To recover these contributions, one needs to carry out calculations to higher loops and to include contributions from higher dimensional operators. Those are much more complicated. In our full one-loop results, we keep all these terms but leave an ambiguity in choosing the value of $\mu$ in $\alpha_{s}(\mu)$. To estimate the uncertainty caused by it, we take three different values of $\mu$. They are $\sqrt{s}, 2 m_{c}$, and $m_{c}$. The corresponding values of $\alpha_{s}(\mu)$ are $\alpha_{s}(\sqrt{s})=0.17, \alpha_{s}\left(2 m_{c}\right)=0.24$, and $\alpha_{s}\left(m_{c}\right)=0.30$.

As for the hadron matrix elements, we will take them as same as those used in [2] [21] [22],

$$
\begin{aligned}
\left\langle 0\left|\chi^{\dagger} \psi\right| \eta_{c}\right\rangle\left\langle\eta_{c}\left|\psi^{\dagger} \chi\right| 0\right\rangle & =0.437 \mathrm{GeV}^{3} \\
\left\langle 0\left|\chi^{\dagger} \psi\right| \eta_{c}(2 S)\right\rangle\left\langle\eta_{c}(2 S)\left|\psi^{\dagger} \chi\right| 0\right\rangle & =0.274 \mathrm{GeV}^{3} \\
\frac{1}{3}\left|\left\langle\chi_{c 0}\left|\psi^{\dagger}\left(-\frac{i}{2} \overleftrightarrow{\mathrm{D}} \cdot \sigma\right) \chi\right| 0\right\rangle\right|^{2} & =0.051 \mathrm{GeV}^{5} \\
\frac{1}{2}\left|\left\langle\chi_{c 1}\left|\psi^{\dagger}\left(-\frac{i}{2} \overleftrightarrow{\mathrm{D}} \times \sigma \cdot \epsilon_{H}\right) \chi\right| 0\right\rangle\right|^{2} & =0.060 \mathrm{GeV}^{5} \\
\mid \sum_{i j}\left\langle\chi_{c 2}\right| \psi^{\dagger}\left(-\frac{i}{2} \overleftrightarrow{D}^{(i} \sigma^{j}\right) & \left.\epsilon_{H}^{i j}\right)\left.\chi|0\rangle\right|^{2}=0.068 \mathrm{GeV}^{5}
\end{aligned}
$$

For the $\eta_{c}$ production, in order to assess the contribution from the relativistic correction, we will evaluate the NRQCD matrix element corresponding to the relativistic correction by using the Gremm-Kapustin[23] relation,

$$
\operatorname{Re}\left\langle 0\left|\chi^{\dagger}\left(-\frac{i}{2} \overleftrightarrow{\mathbf{D}}\right)^{2} \psi\right| \eta_{c}\right\rangle\left\langle\eta_{c}\left|\psi^{\dagger} \chi\right| 0\right\rangle=m^{2} v^{2} \operatorname{Re}\left\langle 0\left|\chi^{\dagger} \psi\right| \eta_{c}\right\rangle\left\langle\eta_{c}\left|\psi^{\dagger} \chi\right| 0\right\rangle
$$

We take $v^{2}=0.13$ for $\eta_{c}$ as that in Ref. [24] and the correspondent NLO NRQCD matrix element yields:

$$
\operatorname{Re}\left\langle 0\left|\chi^{\dagger}\left(-\frac{i}{2} \overleftrightarrow{\mathrm{D}}\right)^{2} \psi\right| \eta_{c}\right\rangle\left\langle\eta_{c}\left|\psi^{\dagger} \chi\right| 0\right\rangle=0.111 \mathrm{GeV}^{5}
$$

For the $\eta_{c}(2 S)$ production, the LO NRQCD matrix element, which is decided by fitting data from $\Gamma\left[\psi(2 S) \rightarrow e^{+} e^{-}\right]$based on heavy-quark spin symmetry, is accurate only in the 
TABLE I: Predicted cross sections (in fb) for the production of $\eta_{c}, \eta_{c}(2 S)$, and $\chi_{c} J$ with various $\mu$. $\sigma^{(0)}$ and $\sigma$ are the cross sections with the LO and the NLO short-distance coefficients, repectively. $\frac{\sigma}{\sigma^{(0)}}-1$ is relative corrections caused by the NLO corrections.

\begin{tabular}{lc|cc|cc|cc}
\hline \hline & \multicolumn{4}{c}{$\mu=\sqrt{s}$} & \multicolumn{2}{c}{$\mu=2 m$} & \multicolumn{2}{c}{$\mu=m$} \\
\hline & $\sigma^{(0)}$ & $\frac{\sigma}{\sigma^{(0)}}-1$ & $\sigma$ & $\frac{\sigma}{\sigma^{(0)}}-1$ & $\sigma$ & $\frac{\sigma}{\sigma^{(0)}}-1$ & $\sigma$ \\
\hline$\eta_{c}$ & 83.3 & $-13.0 \%$ & 72.5 & $-18.4 \%$ & 68.0 & $-23.0 \%$ & 64.1 \\
\hline$\eta_{c}(2 S)$ & 52.2 & $-13.0 \%$ & 45.4 & $-18.4 \%$ & 42.6 & $-23.0 \%$ & 40.2 \\
\hline$\chi_{c 0}$ & 1.19 & $10.4 \%$ & 1.31 & $14.7 \%$ & 1.36 & $18.4 \%$ & 1.41 \\
\hline$\chi_{c 1}$ & 14.3 & $-17.1 \%$ & 11.9 & $-24.1 \%$ & 10.9 & $-30.1 \%$ & 10.0 \\
\hline$\chi_{c 2}$ & 6.28 & $-48.9 \%$ & 3.21 & $-69.0 \%$ & 1.95 & $-86.2 \%$ & 0.87 \\
\hline \hline
\end{tabular}

LO in $v$ as pointed out in Ref. [2]. Therefore we will not consider the relativistic correction to $\eta_{c}(2 S)$ production for consistence.

In the following subsection, we will use these input parameters to calculate the production cross sections.

\section{B. Numerical calculations}

With the parameters given above, we can evaluate the cross sections for the production of $\eta_{c}, \eta_{c}(2 S)$, and $\chi_{c J}$ by using Eqs. (23), (32), (48), and (56). For comparison, we list the results of the LO and the NLO in $\alpha_{s}$ in Table I] For the NLO results, we have taken $\mu$ in $\alpha_{s}(\mu)$ to be $\sqrt{s}, 2 m_{c}$, and $m_{c}$. In Tabel प, we have omitted relativistic corrections. From the table, we see that most of the QCD radiative corrections are negative and considerable. For some processes like $\chi_{2}$ production, the corrections are unexpectedly large.

Then we can estimate the relativistic corrections by Eq. (23). After accounting for the NLO radiative QCD correction(we take $\mu=2 m_{c}$ in $\left.\alpha_{s}(\mu)\right)$ and the relativistic correction, the total cross section of the $\eta_{c}$ production decrease from $83.3 \mathrm{fb}$ to $58.2 \mathrm{fb}$, where the NLO radiative QCD correction and relativistic correction contribute $-15.3 \mathrm{fb}$ and $-9.81 \mathrm{fb}$ respectively. We see that both of them give considerably negative contributions.

These theoretical predictions for the cross sections receive uncertainties from the ambiguities of the values of input parameters. These parameters are the $c$ quark pole mass, 
TABLE II: Predicted cross sections (in $\mathrm{fb}$ ) for the production of $\eta_{c}, \eta_{c}(2 S), \chi_{c J}$ by including theoretical uncertainties.

\begin{tabular}{ccccc}
\hline \hline$\eta_{c}$ & $\eta_{c}(2 S)$ & $\chi_{c 0}$ & $\chi_{c 1}$ & $\chi_{c 2}$ \\
\hline $68.0_{-20.3}^{+22.2}$ & $42.6_{-8.8}^{+10.9}$ & $1.36_{-0.26}^{+0.26}$ & $10.9_{-3.4}^{+3.7}$ & $1.95_{-1.56}^{+1.85}$ \\
\hline \hline
\end{tabular}

the NRQCD matrix elements, and the $\mu$ in $\alpha_{s}(\mu)$. The $c$ quark pole mass may effect both the short-distance coefficients and NRQCD matrix elements. Especially, the matrix element of the relativistic correction term is very sensitive to the value of the pole mass when the Gremm-Kapustin[23] relation (62) is used to estimate its value. For more details, we refer the reader to Ref. [2]. Another theoretical uncertainties arise from the higher order QCD corrections and higher order relativistic corrections. Including these corrections, the $\mu$ dependence and uncertainties of the matrix elements may be reduced. Varying the values of all these parameters in reasonable ranges and omitting higher order correction terms both in $\alpha_{s}$ and in $v$, we can estimate theoretical uncertainties on the predicted cross sections. The results are listed in Table [I.

In $e^{+} e^{-}$collisions, the heavy quarkonium with $C$-parity even can also be produced via the double photon processes. At very high energy limit, this process can be described by the equivalent photon approximation(EPA). Comparing to the processes $e^{+} e^{-} \rightarrow H+\gamma(H=$ $\left.\eta_{c}, \eta_{c}(2 S), \chi_{c J}\right)$, the total production cross sections of the double photon processes are suppressed by additional power of $\alpha$ but enhanced by $\log ^{2} s / m_{e}^{2}[25]$. The cross sections of the $s-$ channel processes $e^{+} e^{-} \rightarrow H+\gamma\left(H=\eta_{c}, \eta_{c}(2 S), \chi_{c J}\right)$ are suppressed by $M_{h}^{4} / s^{2}$. Combining all these factors, at the $\mathrm{B}$ factories, the production cross sections of the heavy quarkonium with $C$-parity even via the double photon process are larger than that via associated production with photon. However, the signals in detectors from these two production mechanisms are very different. Especially, the produced heavy quarkonia via double photon processes are predominated by small $P_{T}$ events at high energy which are hard to be detected from their decay products. On the other hand, the produced heavy quarkonia via associated production with photon are insensitive to the $P_{T}$. Moreover, the events can be reconstructed by measuring the photon assuming the photon detector is good enough. A thorough analysis on these processes in the framework of the NRQCD factorization formalism will be presented elsewhere. 


\section{Acknowledgments}

After this work was finished, we were told that D. Li, Z.G. He, and K.T. Chao had done a similar work [26]. One of the authors, Wen-Long Sang would like to thank G. T. Bodwin and Yu Jia for useful discussion and to thank Zhi-Guo He for checking results. This work was supported partly by the National Natural Science Foundation of China (NNSFC) under No.10875156.

\section{Appendix A: The NLO radiative corrections to short distance coefficients for the} $\eta_{c}$ production

The definition for $C(r)$ appeared in Eq. (28):

$$
\begin{aligned}
C(r) & =-\frac{2\left[30 r^{2}-\left(84+\pi^{2}\right) r+2 \pi^{2}+54\right]}{9(2-r)(1-r)}+\frac{8(2 r-3)}{3(2-r)^{2}} \log \left(\frac{2}{r}-2\right)-\frac{4}{b} \log \left(\frac{1-b}{1+b}\right) \\
& +\frac{2}{3(r-1)}\left[\left(1+\frac{r}{2}\right) \log ^{2}\left(\frac{1-b}{1+b}\right)-\log ^{2}\left(\frac{2}{r}-1\right)\right]+\frac{4}{3(1-r)} \operatorname{Li}_{2}\left(\frac{r}{2-r}\right),
\end{aligned}
$$

where we use $r=\frac{4 m^{2}}{s}, b=\sqrt{1-4 m^{2} / s}$ and $m=m_{c}$ in Eq. (B1). In addition, dilogarithm $\left(\operatorname{Li}_{2}(x)\right)$ is used in the Appendices $\mathrm{A}$ and $\mathrm{B}$, which is defined as

$$
\mathrm{Li}_{2} \equiv-\int_{0}^{x} \frac{d z}{z} \log (1-z)
$$

where $x \leq 1$.

The definition for $C^{\prime}(\mathrm{r})$ appeared in Eq. (34):

$$
\begin{aligned}
C^{\prime}(r) & =C_{F} \frac{\pi^{2}(r-2)(3 r+4)-12(r-1)(5 r-9)}{12(r-1)(r-2)}+C_{F} \frac{2(2 r-3)}{(r-2)^{2}} \log \left(2-\frac{2}{r}\right) \\
& +\frac{C_{F}}{r-1}\left\{\operatorname{Li}_{2}\left(\frac{2}{r}-1\right)-\tan ^{-1}\left(\frac{1}{\sqrt{r-1}}\right)[6 \sqrt{(r-1)}\right. \\
& \left.\left.+(r+2) \tan ^{-1}\left(\frac{1}{\sqrt{r-1}}\right)\right]\right\},
\end{aligned}
$$


Appendix B: The NLO radiative corrections to short distance coefficients for the $\chi_{J}$ production

1. $\chi_{0}$

$$
\begin{aligned}
C_{0}^{0}(r) & =\frac{-2}{9\left(3 r^{3}-r^{2}+3 r-1\right)}\left[\frac{\pi^{2}\left(4 r^{4}-7 r^{3}+11 r^{2}-r+1\right)}{(1-r)^{2}}\right. \\
& \left.+\frac{6 r\left(7 r^{4}-25 r^{3}+31 r^{2}-25 r+24\right)}{(2-r)^{2}}\right] \\
& +\frac{1}{3(r-1)^{2}(3 r-1)}\left\{\frac{2\left(4 r^{4}-7 r^{3}+11 r^{2}-r+1\right) \log ^{2}\left(\frac{2}{r}-1\right)}{1+r^{2}}\right. \\
& +\left(3 r^{3}-6 r^{2}-3 r+2\right) \log ^{2}\left(\frac{1-b}{1+b}\right)+\frac{4(1-r)}{b}\left(3 r^{2}-12 r+5\right) \log \left(\frac{1-b}{1+b}\right) \\
& -\frac{8\left(2 r^{4}-5 r^{3}+6 r^{2}-2 r+1\right) \log \left(\frac{2}{r}-2\right) \log \left(\frac{2}{r}-1\right)}{\left(1+r^{2}\right)} \\
& \left.+\frac{8\left(8 r^{5}-50 r^{4}+125 r^{3}-152 r^{2}+87 r-18\right) \log \left(\frac{2}{r}-2\right)}{(r-2)^{3}}\right\} . \\
& +\frac{4\left[\left(4 r^{4}-7 r^{3}+11 r^{2}-r+1\right) \operatorname{Li}_{2}\left(\frac{r}{2-r}\right)-2\left(2 r^{4}-5 r^{3}+6 r^{2}-2 r+1\right) \operatorname{Li}_{2}\left(2-\frac{2}{3}\right)\right.}{3(3 r-1)(1-r)^{2}\left(1+r^{2}\right)}
\end{aligned}
$$

2. $\chi_{1}$

$$
\begin{aligned}
C_{1}^{ \pm 1}(r) & =\frac{\pi^{2}\left(-4 r^{2}+19 r-5\right)-36(r-1)^{2}}{9(r-1)^{2}}+\frac{\left(-3 r^{2}+3 r-2\right) \log ^{2}\left(\frac{1-b}{1+b}\right)}{3(r-1)^{2}} \\
& +\frac{4\left[(r-2)\left(2 r^{2}-r+1\right) \log \left(\frac{1-b}{1+b}\right)+b\left(2 r^{2}-5 r+1\right) \log \left(\frac{2}{r}-2\right)\right]}{3 b(r-2)(r-1)} \\
& +\frac{\left[\left(4 r^{2}-19 r+5\right) \log \left(\frac{2}{r}-1\right)-2\left(r^{2}-16 r+3\right) \log \left(\frac{2}{r}-2\right)\right] \log \left(\frac{2}{r}-1\right)}{3(r-1)^{2}} \\
& +\frac{2\left(4 r^{2}-19 r+5\right) \operatorname{Li}_{2}\left(\frac{r}{2-r}\right)-2\left(r^{2}-16 r+3\right) \operatorname{Li}_{2}\left(2-\frac{2}{r}\right)}{3(r-1)^{2}} .
\end{aligned}
$$




$$
\begin{aligned}
C_{1}^{0}(r) & =\frac{2\left[\pi^{2}(r-2)\left(r^{2}-r+2\right)-6(r-1)(4 r-7)\right]}{9(r-1)(r-2)}+\frac{4\left(r^{2}-2 r-1\right) \log \left(\frac{1-b}{1+b}\right)}{3 b(1-r)} \\
& +\frac{8\left(3 r^{3}-10 r^{2}+9 r-1\right) \log \left(\frac{2}{r}-2\right)}{3(r-2)^{2}(r-1)}+\frac{(r-2)\left(r^{2}+1\right) \log ^{2}\left(\frac{1-b}{1+b}\right)}{3(r-1)^{2}} \\
& -\frac{2 \log \left(\frac{2}{r}-1\right)\left[2(1-2 r) \log \left(\frac{2}{r}-2\right)+(r-1)\left(r^{2}-r+2\right) \log \left(\frac{2}{r}-1\right)\right]}{3(r-1)^{2}} \\
& -\frac{4\left[(1-2 r) \operatorname{Li}_{2}\left(2-\frac{2}{r}\right)+(r-1)\left(r^{2}-r+2\right) \operatorname{Li}_{2}\left(\frac{r}{2-r}\right)\right]}{3(r-1)^{2}} .
\end{aligned}
$$

3. $\chi_{2}$

$$
\begin{aligned}
C_{2}^{ \pm 2}(r)= & -\frac{2 r^{2}\left[24(r-1)^{2}+\pi^{2}(r+3)\right]}{9(r-1)^{2}}+\frac{8 r^{2}\left[4 b \log \left(\frac{2}{r}-2\right)-(r-5) \log \left(\frac{1-b}{b+1}\right)\right]}{3 b(r-1)} \\
+ & \frac{2 r^{2}\left\{\log \left(\frac{2}{r}-1\right)\left[(r+3) \log \left(\frac{2}{r}-1\right)-2(r-1) \log \left(\frac{2}{r}-2\right)\right]-4 \log ^{2}\left(\frac{1-b}{b+1}\right)\right\}}{3(r-1)^{2}} \\
+ & \frac{4 r^{2}\left[(r+3) \operatorname{Li}_{2}\left(\frac{r}{2-r}\right)-(r-1) \operatorname{Li}_{2}\left(2-\frac{2}{r}\right)\right]}{3(r-1)^{2}}, \\
C_{2}^{ \pm 1}(r)= & \frac{\pi^{2}(2-r)\left(2 r^{2}-20 r+3\right)-12 r(r-1)^{2}}{9(r-2)(r-1)^{2}}+\frac{\left(3 r^{2}+21 r-2\right) \log ^{2}\left(\frac{1-b}{b+1}\right)}{3(r-1)^{2}} \\
& +\frac{\log \left(\frac{2}{r}-1\right)\left[\left(2 r^{2}-20 r+3\right) \log \left(\frac{2}{r}-1\right)-2\left(5 r^{2}+r+1\right) \log \left(\frac{2}{r}-2\right)\right]}{3(r-1)^{2}} \\
& -\frac{4\left[(19 r+3)(r-2)^{2} \log \left(\frac{1-b}{1+b}\right)+b\left(10 r^{3}-49 r^{2}+51 r+10\right) \log \left(\frac{2}{r}-2\right)\right]}{3 b(r-2)^{2}(r-1)} \\
& -\frac{2\left[\left(5 r^{2}+r+1\right) \operatorname{Li}_{2}\left(2-\frac{2}{r}\right)-\left(2 r^{2}-20 r+3\right) \operatorname{Li}_{2}\left(\frac{r}{2-r}\right)\right]}{3(r-1)^{2}},
\end{aligned}
$$




$$
\begin{aligned}
C_{2}^{0}(r) & =-\frac{\pi^{2}\left(2 r^{4}+9 r^{3}+27 r^{2}-11 r+5\right)(r-2)^{2}+12(r-1)^{2}\left(2 r^{3}-11 r^{2}+6 r+12\right)}{9(r-2)^{2}(r-1)^{2}} \\
& -\frac{\left(3 r^{3}+30 r^{2}+3 r+2\right) \log ^{2}\left(\frac{1-b}{1+b}\right)}{3(r-1)^{2}}+\frac{\left(2 r^{4}+9 r^{3}+27 r^{2}-11 r+5\right) \log ^{2}\left(\frac{2}{r}-1\right)}{3(r-1)^{2}} \\
& +\frac{8\left(6 r^{5}-38 r^{4}+60 r^{3}+16 r^{2}-69 r+6\right) \log \left(\frac{2}{r}-2\right)}{3(r-2)^{3}(r-1)}+\frac{4\left(21 r^{2}+18 r-1\right) \log \left(\frac{1-b}{1+b}\right)}{3 b(r-1)} \\
& -\frac{2\left(2 r^{4}+9 r^{3}-9 r^{2}-11 r+3\right) \log \left(\frac{2}{r}-2\right) \log \left(\frac{2}{r}-1\right)}{3(r-1)^{2}} \\
& +\frac{2}{3(r-1)^{2}}\left[\left(-2 r^{4}-9 r^{3}+9 r^{2}+11 r-3\right) \operatorname{Li}_{2}\left(2-\frac{2}{r}\right)\right. \\
& \left.+\left(2 r^{4}+9 r^{3}+27 r^{2}-11 r+5\right) \operatorname{Li}_{2}\left(\frac{r}{2-r}\right)\right] .
\end{aligned}
$$

\section{4. the expressions for $C_{J}^{\prime}(r)$}

For $C_{0}^{\prime}(r)$ :

$$
\begin{aligned}
C_{0}^{\prime}(r) & =\frac{C_{F}}{6(r-2)^{2}(r-1)^{2}(3 r-1)}\left[-6 r\left(7 r^{2}-25 r+24\right)(r-1)^{2}\right. \\
& \left.+\pi^{2}(r-2)^{2}(3 r-1)-6 \pi(r-2)^{2} \sqrt{r-1}\left(3 r^{2}-12 r+5\right)\right] \\
& +\frac{2 C_{F}(2 r-3)\left(4 r^{3}-15 r^{2}+19 r-6\right) \log \left(2-\frac{2}{r}\right)}{(r-2)^{3}(r-1)(3 r-1)}-\frac{C_{F} \operatorname{Li}_{2}\left(\frac{2}{r}-1\right)}{(r-1)^{2}} \\
& +\frac{C_{F}}{(r-1)^{2}(3 r-1)} \tan ^{-1}(\sqrt{r-1})\left[2 \sqrt{r-1}\left(3 r^{2}-12 r+5\right)\right. \\
& \left.+\pi\left(3 r^{3}-6 r^{2}-3 r+2\right)-\left(3 r^{3}-6 r^{2}-3 r+2\right) \tan ^{-1}(\sqrt{r-1})\right] .
\end{aligned}
$$

For $C_{1}^{\prime}(r)$ :

$$
\begin{aligned}
C_{1}^{\prime}(r) & =\frac{C_{F}}{12(r-2)(r-1)^{2}(r+1)}\left[-12(r-1)^{2}\left(3 r^{2}-2 r-7\right)\right. \\
& \left.+\pi^{2}(r-2)\left(r^{3}+r^{2}+2\right)+12 \pi \sqrt{r-1}(r-2)\left(2 r^{3}-2 r^{2}+3 r+1\right)\right] \\
& +\frac{C_{F}\left(2 r^{4}-3 r^{3}-9 r^{2}+16 r-2\right) \log \left(2-\frac{2}{r}\right)}{(r-2)^{2}\left(r^{2}-1\right)}-\frac{C_{F}\left(r^{3}+r^{2}+2\right) \operatorname{Li}_{2}\left(\frac{2}{r}-1\right)}{2(r-1)^{2}(r+1)} \\
& +\frac{C_{F}}{(r-1)^{2}(r+1)} \tan ^{-1}(\sqrt{r-1})\left[-2 \sqrt{r-1}\left(2 r^{3}-2 r^{2}+3 r+1\right)\right. \\
& \left.-\pi\left(2 r^{3}-r^{2}+r+2\right)+\left(2 r^{3}-r^{2}+r+2\right) \tan ^{-1}(\sqrt{r-1})\right] .
\end{aligned}
$$


For $C_{2}^{\prime}(r)$ :

$$
\begin{aligned}
C_{2}^{\prime}(r) & =\frac{-C_{F}}{12(r-2)^{2}(r-1)^{2}\left(6 r^{2}+3 r+1\right)}\left[12\left(24 r^{4}-91 r^{3}+79 r^{2}+6 r+12\right)(r-1)^{2}\right. \\
& \left.+\pi^{2}(r-2)^{2}\left(9 r^{3}-21 r^{2}-6 r-2\right)+12 \pi \sqrt{r-1}(r-2)^{2}\left(12 r^{3}-24 r^{2}-9 r+1\right)\right] \\
& +\frac{C_{F}\left(30 r^{5}-157 r^{4}+249 r^{3}-76 r^{2}-78 r+12\right) \log \left(2-\frac{2}{r}\right)}{(r-2)^{3}(r-1)\left(6 r^{2}+3 r+1\right)} \\
& +\frac{C_{F}\left(9 r^{3}-21 r^{2}-6 r-2\right) \operatorname{Li}_{2}\left(\frac{2}{r}-1\right)}{2(r-1)^{2}\left(6 r^{2}+3 r+1\right)} \\
& -\frac{C_{F}}{(r-1)^{2}\left(6 r^{2}+3 r+1\right)} \tan ^{-1}(\sqrt{r-1})\left[-2 \sqrt{r-1}\left(12 r^{3}-24 r^{2}-9 r+1\right)\right. \\
& \left.+\pi\left(-6 r^{3}+15 r^{2}+9 r+2\right)+\left(6 r^{3}-15 r^{2}-9 r-2\right) \tan ^{-1}(\sqrt{r-1})\right]
\end{aligned}
$$

[1] G. T. Bodwin, E. Braaten, and G. P. Lepage, Phys. Rev. D 51, 1125 (1995) [Erratum-ibid. D 55, 5853 (1997)] arXiv:hep-ph/9407339.

[2] H. S. Chung, J. Lee, and C. Yu, Phys. Rev. D78, 074022,(2008) arXiv:hep-ph/08081625]

[3] B. Guberina, J. H. Kuhn, R. D. Peccei and R. Ruckl, Nucl. Phys. B 174, 317 (1980).

[4] M. A. Shifman and M. I. Vysotsky, Nucl. Phys. B 186, 475 (1981).

[5] G. T. Bodwin and A. Petrelli, Phys. Rev. D 66, 094011 (2002) arXiv:hep-ph/0205210.

[6] A. Czarnecki and K. Melnikov, Phys. Rev. Lett 80, 2531 (1998) arXiv:hep-ph/9712222.

[7] M. Beneke, A. Signer and V. A. Smirnov, Phys. Rev. Lett 80, 2535 (1998) arXiv:hep-ph/9712302.

[8] G. T. Bodwin, H. S. Chung, J. Lee and C. Yu, Phys. Rev. D 79, 014007 (2009) arXiv:0807.2634].

[9] G. T. Bodwin and J. Lee, Phys. Rev. D 69, 054003(2004).

[10] E. Braaten and Y. Q .Chen, Phys. Rev. D 57, 4236-4253 (1998) [Erratum-ibid. D 59, 079901 (1999)] arXiv:hep-ph/9710357.

[11] Y. J. Zhang and K. T. Chao, Phys. Rev. D 78, 094017 (2008) arXiv:0808.2985.

[12] R. Mertig, M. Böhm and A. Denner, Comput. Phys. Commun. 64, 345 (1991)

[13] G. 'T. Hooft and M. Veltman, Nuclear. Physics. B 153, 365-401 (1979)

[14] R. Keith. Ellis and Giulia. Zanderighi, JHEP 0802:002 (2008) arXiv:0712.1851. 
[15] Y. Jia and D. Yang, Nucl. Phys. B 814, 217-230 (2009) arXiv:0812.1965].

[16] A. Petrelli, M. Cacciari, M. Greco, F. Maltoni and M. L. Mangano, Nucl. Phys. B. 514, 245-309 (1998)

[17] E. Braaten and J. Lee, Phys. Rev. D 67, 054007 (2003) [Erratum-ibid. D 72, 099901 (2005)] arXiv:hep-ph/0211085.

[18] E. Braaten and Y. Q. Chen, Phys. Rev. Lett 76, 730-733 (1996) arXiv:hep-ph/9508373.

[19] R. Barbieri, R. Gatto, R. Kögerler and Z. Kunszt, Phys. Lett. 57B, 455 (1975).

[20] W. Celmaster, Phys. Rev. D 19, 1517 (1979).

[21] G. T. Bodwin, H. S. Chung, D. Kang, J. Lee, and C. Yu, Phys. Rev. D 77, 094017 (2008) arXiv:0710.0994.

[22] G. T. Bodwin, D. Kang and J. Lee, Phys. Rev. D 74, 014014 (2006) arXiv:hep-ph/0603186].

[23] M. Gremm and A. Kapustin, Phys. Lett. B 407, 323 (1997) arXiv:hep-ph/9701353.

[24] W. L. Sang, L. F. Yang and Y. Q. Chen, Phys. Rev. D 80, 014013 (2009).

[25] S. J. Brodsky, T. Kinoshita and H. Terazawa, Phys. Rev. Lett 25, 972-975 (1970).

[26] D. Li, Z. G. He, K. T. Chao, Phys. Rev. D 80, 114014 (2009) arXiv:0910.4155] 